\title{
Search for Invisible Higgs Bosons Produced via Vector Boson Fusion at the LHC Using ATLAS Detector
}

\author{
Mohamed Zaazoua, ${ }^{1}$ Farida Fassi ${ }^{1}$, Kétévi Adiklè Assamagan ${ }^{2}$, and Loan Truong \\ ${ }^{1}$ Mohammed $V$ University in Rabat, Morocco \\ ${ }^{2}$ Brookhaven National Laboratory, USA
}

\begin{abstract}
A variety of astrophysical observations showed direct evidence for the existence of dark matter which accounts for about $85 \%$ of matter in the universe and does not interact with ordinary matter, except through gravity. Despite its abundance, dark matter particles are very elusive and hard to spot and no experiment confirmed their existence. In this work the invisible Higgs sector was investigated where Higgs bosons are produced via the vector boson fusion (VBF) process and subsequently decay into invisible particles. The expectation for the branching fraction of invisible decays from the standard model is $O(0.1) \%$ but several scenarios beyond the standard model allow larger values of $O(10) \%$. The hypothesis under consideration is that the Higgs boson might decay into a pair of weakly interacting massive particles (WIMPs) which are candidates to explain the existence of dark matter. The experimental signature in the detector is a pair of energetic jets and large missing energy. The analysis uses data samples of an integrated luminosity of 139 $\mathrm{fb}^{-1}$ of proton proton collisions at $\sqrt{s}=13 \mathrm{TeV}$ recorded by ATLAS detector at the LHC. The observed number of events are found to be in agreement with the background expectation from standard model processes. Assuming a $125 \mathrm{GeV}$ Higgs boson with a standard model production cross section, the observed and expected upper limits on the branching fraction of its decay into invisible particles are found to be 0.13 at $95 \%$ confidence level.
\end{abstract}

Keywords: invisible, Higgs, dark matter, decay, VBF, WIMPs

DOI: 10.31526/ACP.BSM-2021.10

\section{MOTIVATION}

Based on many astrophysical observations, there are strong evidences about the existence of dark matter (DM) which make up most of matter in the universe [1] (see Fig 1). The visible matter make only $5 \%$ of the universe, and the rest is dark matter (27\%) and dark energy (68\%). Yet DM is completely invisible to traditional detectors, we can only detect it from its gravitational effects.

Searches for DM are gaining increasing importance at the Large Hadron Collider (LHC). One of the promising tools is through the invisible decays of the Higgs boson. Where the Higgs could be a mediator between Standard Model (SM) particles and ones that belong to the DM sector. Fig 1 shows the possible DM detection channels.

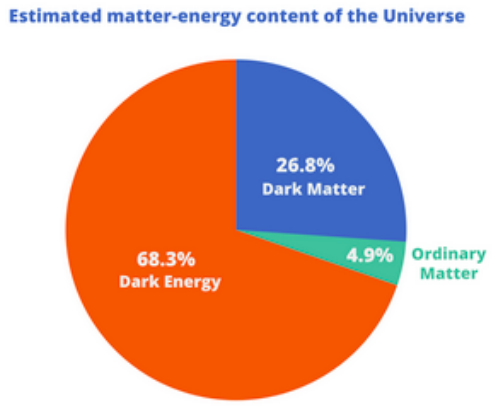

QATLAS

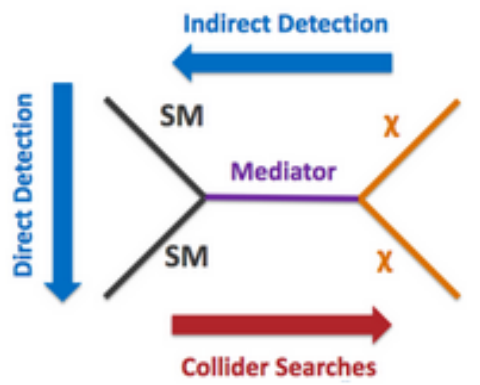

Collider Searches

FIGURE 1: Estimated DM - visible matter in the universe and the possible DM detection channels

\section{INTRODUCTION}

The Analysis uses data samples produced with a luminosity of $139 \mathrm{fb}^{-1}$ of proton-proton collisions at center of mass of $\sqrt{s}=13$ $\mathrm{TeV}$, recorded by the ATLAS detector at the LHC. The SM expects that no more than $0.1 \%$ of the branching fraction $\left(B_{H \rightarrow i n v}\right)$ of the Higgs bosons will decay into invisible particles [2]. Other Beyond Standard Model (BSM) scenarios expect larger values for 
the invisible Higgs branching ratio, up to $10 \%$ [3]. We are considering the scenario where the Higgs is produced via Vector Boson Fusion (VBF) mechanism and decays into a pair of weakly interacting massive particles(WIMPs) that are candidates to explain the existence of dark matter. A Feynman diagram schematic of the signal like events is shown in Fig 2

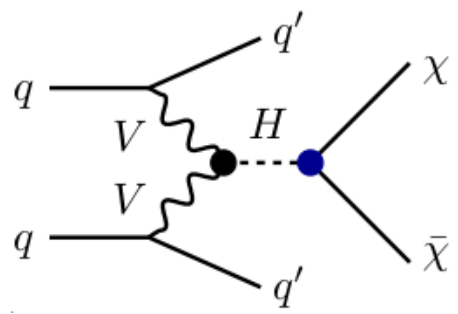

FIGURE 2: Feynman diagram of a signal region like event where a Higgs bonson is produced via VBF and decay invisibly into a pair of WIMPs.

The VBF topology offers a powerful rejection against background from single vector boson production. the contribution of gluon-gluon fusion ( $\mathrm{ggF}$ ) process in the signal region (SR) is tiny compared to the VBF process and it is also considered as part of the signal.

The experimental signature from VBF process is two leading jets with large values on the difference of their pseudo-rapidity $\left(\Delta \eta_{j j}\right)$ and large invariant masses $m_{j j}$, because the two jet lies in opposite hemisphere of the detector and more forward than other jets from non VBF processes.

The previous version of the analysis set up a limit on the $B_{H \rightarrow i n v}$ of 0.37 at $95 \%$ confidence level (CL) [5]. In this analysis many changes and improvements were added, selections criteria on mjj, $\Delta \eta_{j j}$ and $\Delta \Phi_{j j}$ are relaxed while the missing transverse energy $\left(E_{T}^{\text {miss }}\right)$ is slightly increased. the current analysis consider also events with three or four jets. To benefit from the large data set, a further binning on $m_{j j}$ is applied and for the first time a binning on $\Delta \Phi_{j j}$ and the jet multiplicity. Fig 3 shows a view of eleven bins in the signal region. These changes yields to a better signal to background ratio in the region of large $m_{j j}$ and smaller values of $\Delta \Phi_{j j}$.

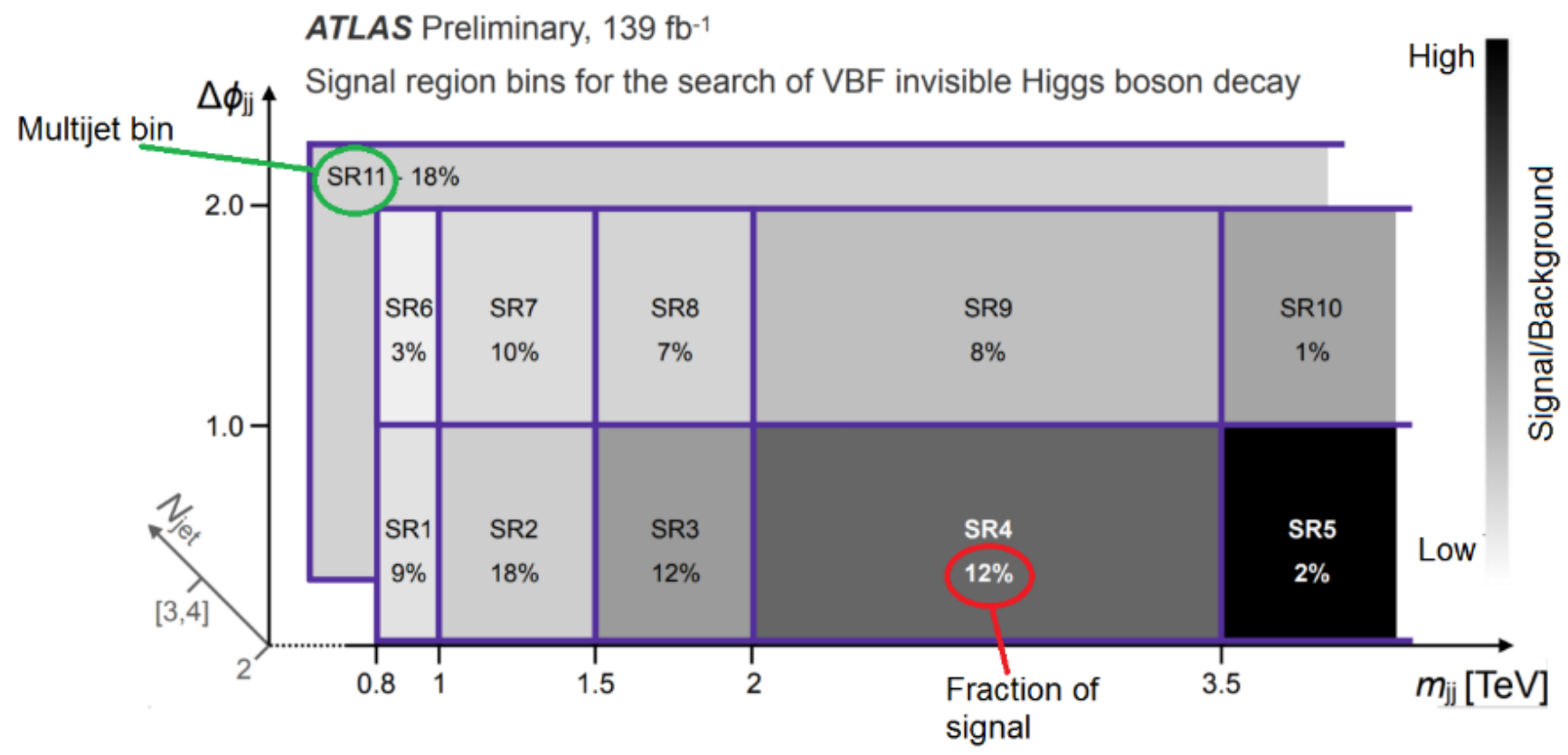

FIGURE 3: The analysis binning strategy, view of the eleven bins in the signal region.

\section{SIGNAL AND CONTROL REGION DEFINITIONS}

The signal and control regions are defined using a set of cuts on the four leading jets, the leptons and the missing transverse energy $\left(E_{T}^{\text {miss }}\right)$.

\subsection{Signal region}

Only events satisfying the following set of criteria will enter the signal region: 
- No lepton or photon candidates in the event.

- The first leading jet has $p_{T}\left(j_{1}\right)>80 \mathrm{GeV}$ and the second leading jet has $p_{T}\left(j_{2}\right)>50 \mathrm{GeV}$. Both steaming from hard scattering process.

- The two leading jets are well separated in $\eta: \Delta \eta_{j j}>3.8$ and lying in opposite hemispheres: $\eta_{1} \cdot \eta_{2}<3.8$, and not back to back $\Delta \Phi_{j j}<2.0$.

- Large invariant mass of the two leading jets: $m j j>0.8 \mathrm{TeV}$

- The third and forth jets have $p_{T}>20 \mathrm{GeV}$. and no more than one of the four jets are b-tagged.

- Centrality of the third and fourth jets $\left(C_{i}\right)<0.6$ and the invariant mass of the extra jet with one of the two leading jets $<2.0$.

- $E_{T}^{\text {miss }}>200 \mathrm{GeV}$ with a soft term less than $20 \mathrm{GeV}$ and the vectorial sum of transverse momentum of all jets $H_{T}^{\text {miss }}>180 \mathrm{GeV}$ to suppress the multijet background.

The events passing these selections are then split using the binning strategy in Fig 3

\subsection{Control regions}

The Analysis define two control regions to estimate the $\mathrm{V}+$ jets background, $\mathrm{Z}(\rightarrow l l)+$ jets control region is used to estimate $\mathrm{Z}(\rightarrow v v)$ + jets in the signal region. Events satisfying the following selections will enter this control region:

- Events have exactly two leptons with $p_{T}>20 \mathrm{GeV}$ for the first leading one.

- The invariant mass of the leptons is close to the mass of $\mathrm{Z}$ boson: $|m l l-m Z|<25 \mathrm{GeV}$.

- The missing transverse energy in the event is less than $70 \mathrm{GeV}$.

A second $\mathrm{W}(\rightarrow l v)$ control region is defined to estimate the contribution of events with a lost lepton $\mathrm{W}\left(\rightarrow l_{\text {lost }} v\right)$ in the signal region. Event entering this control region should satisfy the following criteria.

- Events have exactly one lepton with $p_{T}(l 1)>30 \mathrm{GeV}$.

- $E_{T}^{\text {miss }}$ significance with the fake electron $>4 \sqrt{\mathrm{GeV}}$

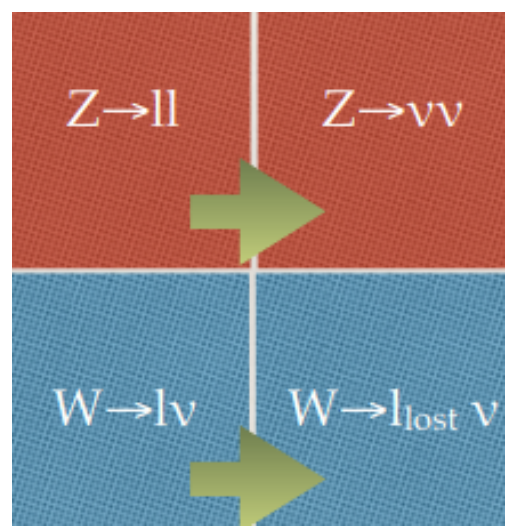

FIGURE 4: $Z(\rightarrow l l)$ control region used to estimate contribution from $Z(\rightarrow v v)$ in SR and the $\mathrm{W}(\rightarrow l v)$ is used to estimate background from $\mathrm{W}\left(\rightarrow l_{\text {lost }} v\right)$ in the SR.

\section{BACKGROUND ESTIMATION}

The $\mathrm{V}+$ jets events represent the dominant background $\sim 95 \%$ in the SR. There are other less important sources of backgrounds like the diboson, $t \bar{t}$ and the multijet (MJ) processes. 

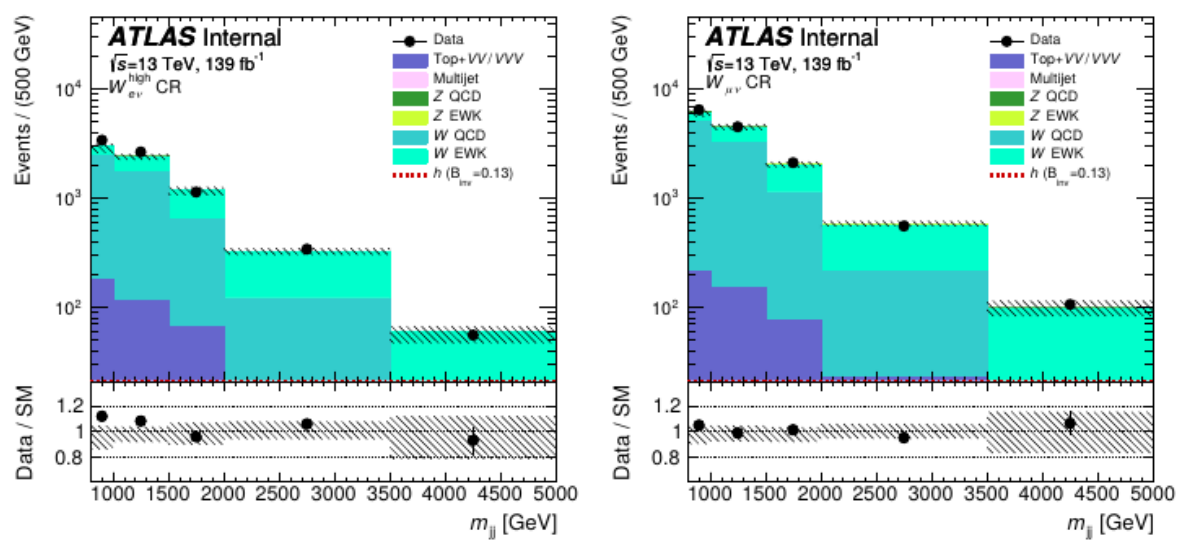

FIGURE 5: $m_{j j}$ distribution in $W_{e v}^{h i g h}$ and $W_{e v}^{l o w}$ control regions. The statistical and reconstruction systematic uncertainties are presented with the hashed band.
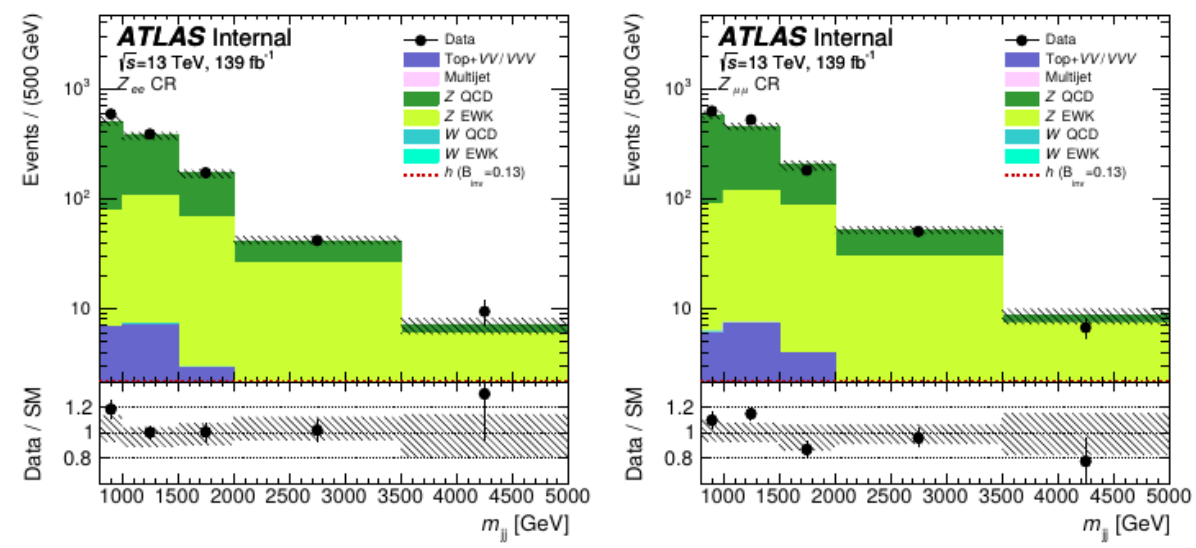

FIGURE 6: $m_{j j}$ distribution in $Z_{e e}$ and $Z_{\mu \mu}$ control regions. The statistical and reconstruction systematic uncertainties are presented with the hashed band.

\subsection{V+jets backgrounds}

To constrain the $\mathrm{V}+$ jets backgrounds, the analysis uses a data driven technique that uses $\mathrm{W}$ and $\mathrm{Z}$ control regions that are divided into 11 bins corresponding to the SR bins in Fig 3 In both cases of W and Z CR, the simulation is normalised to cross section times luminosity in Fig 5 and Fig 6 .

The W CR $\left(W_{e v}^{h l g h}\right)$ can have small contribution from events with fake electron originating from multijet processes originating from an overlap between a jet and an electron. To estimate this contribution, the analysis define a further $W_{e v}^{\text {low }} \mathrm{CR}$ in which enter the events that fail the $E_{T}^{\text {miss }}$ significance cut, with a loose identification requirement for the electron. The ratio of data events with high $E_{T}^{\text {miss }}$ significance to the ones with low $E_{T}^{\text {miss }}$ significance is evaluated in Fig 7 and used to scale the yield of the $W_{e v}^{\text {low }} \mathrm{CR}$. Then events with real electron from simulation are subtracted to get the contribution in the $W_{e v}^{\text {high }}$ CR.
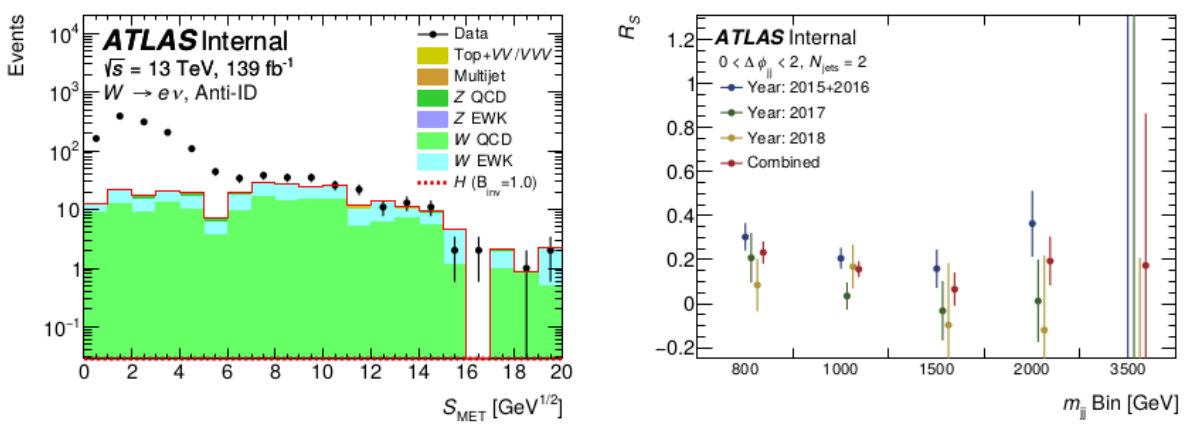

FIGURE 7: Comparison of data and simulation in $W_{e v}^{\text {low }}$ control region, plotted inclusively in $\Delta \Phi_{j j}$ and $m_{j j}$ (left), ratio of number of events in the $W_{e v}^{\text {high }}$ CR to those in the $W_{e v}^{\text {low }}$ CR as a function of $m_{j j}$ for three years of data-taking $(2016,2017$, and 2018) separately (right). 


\subsection{Multijet background}

Events from multijet background are characterised with a large $\Delta \Phi_{j j}$ between the two leading jets and a small values of $E_{T}^{m i s s}$ originating from jet-mismeasurements that are dominated by the jet energy scale (JER). Its contribution is estimated from data using rebalance and smear (R\&S) technique. The method uses an inclusive jet sample recorded by single jet trigger as input, then jets get classified as hard scatter (HS) jet or pileup (PU) jet.

After rebalancing and smearing, events are split into events with HS jet that accumulate at large $\Delta \Phi_{j j}$ or ones with HS+PU jets that accumulate at low $\Delta \Phi_{j j}$. Then both normalised to fit $\Delta \Phi_{j j}$ distribution in loose multijet CR. A correction is applied to account for the inefficiency of $E_{T}^{\text {miss }}$ triggers (see Fig 8 . Performance of the method is validated in low $E_{T}^{m i s s}$ and low $m_{j j} C R$. Fig 9 shows the agreement between the data and the simulation.
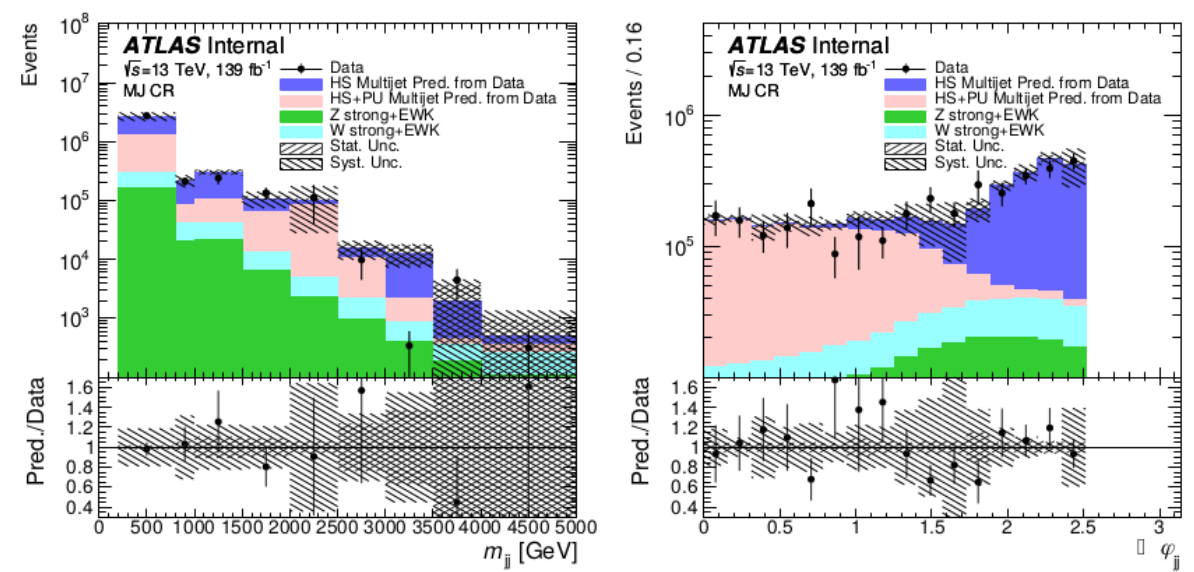

FIGURE 8: $m_{j j}$ and $\Delta \Phi_{j j}$ distribution in the summed MJ CR. The two templates (HS, blue) and (HS+PU, red) are normalised to fit the $\Delta \Phi_{j j}$ distribution.
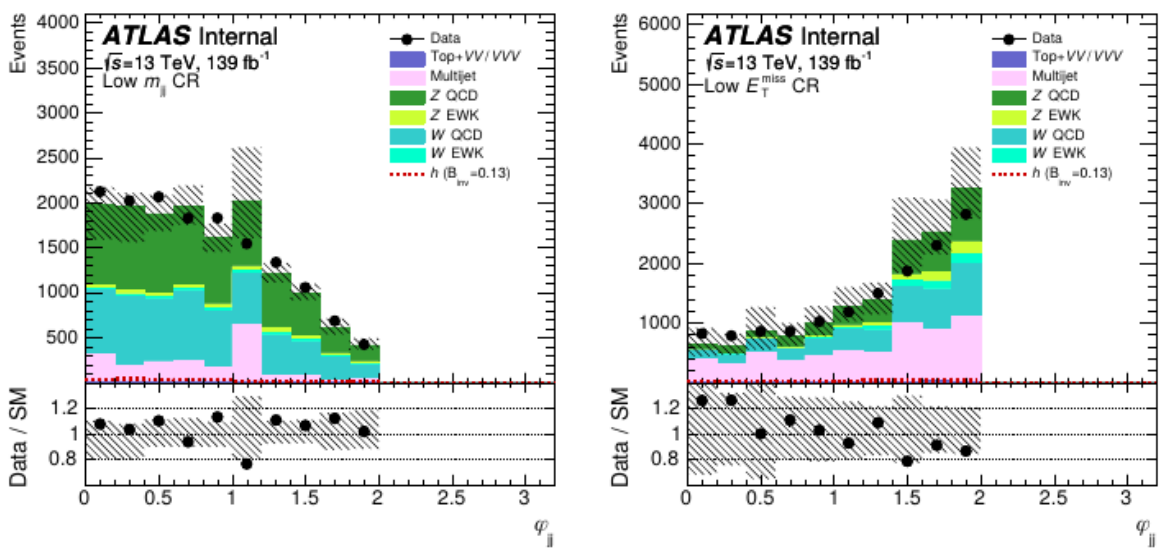

FIGURE 9: $\Delta \Phi_{j j}$ distribution in the summed low $m_{j j}$ (left) and low $E_{T}^{m i s s}$ (right) control regions.

\section{SYSTEMATIC UNCERTAINTIES}

\subsection{Theoretical uncertainties}

The high-order matrix element effects and parton shower matching uncertainties are evaluated using the renormalisation, factorisation, resumation (qsf) and ckkw matching scales.

The renormalisation and factorisation scales are varied up and down by a factor of two, using on-the-fly varied event weights in the Sherpa MC samples. For strong V+jets, downward uncertainties range from 18 to $26 \%$, and the upward uncertainties range from $27 \%$ to $43 \%$. For electroweak V+jets, downward uncertainties range from $9 \%$ to $20 \%$, and the upward uncertainties range from $11 \%$ to $29 \%$.

The uncertainties calculated as relative error, vary from $4 \%$ to $8 \%$ for the qsf, and from $4 \%$ to $6 \%$ for CKKW matching scales. The PDF uncertainties defined as the standard deviation of the ensemble of 100 PDFs within the NNPDF set, range from $1 \%$ to $2 \%$.

For the VBF signal, uncertainties for renormalisation and factorisation scales vary from 1 to $3 \%$, from $2 \%$ to $4 \%$ for the parton shower and from 1 to $2 \%$ for the PDF from NNDF set. 
For the ggF signal, using renormalisation and factorisation scales, uncertainties are evaluated to be $45 \%$ in the 2 -jet bin and $41 \%$ in the 3-jet and 4-jet bins.

\subsection{Experimental uncertainties}

Three main categories of experimental uncertainties are affecting the sensitivity of the analysis. Uncertainty on the luminosity is evaluated to $1,7 \%$ and it effects only the signal yield while it cancels on the background. Uncertainty on the trigger efficiency is evaluated to account for possible trigger efficiency differences between data and simulation, the corresponding value is $2 \%$. The analysis consider also the uncertainties related to the used physics objects and propagate them to the $E_{T}^{\text {miss }}$ calculations.

\section{RESULTS}

The event yields in the eleven bins of all the signal and control regions after the likelihood fit are compared to the observed number of events and found to be in a good agreement with the expected yields from the standard model background (see Fig 10 . Since no excess found above the SM background, a limit setting on the invisible Higgs branching fraction gives a more stringent value of $13 \%$ at $95 \%$ confidence level (CL). This result is interpreted as a search for invisible decays of heavy scalar particles acting as mediator to dark matter particles. Fig 11 shows the product of the cross section and the branching ratio to invisible particles in the range from $50 \mathrm{GeV}$ to $1 \mathrm{TeV}$. The derived limit improves at high masses because of the accumulation of signal in high $m_{j j}$ SR bins.

The overlay in Fig 12 shows the interpretation of the limit on the spin-independent WIMP-nucleon cross section for the Higgs portal models compared to results from direct detection experiments. A limit of $11 \%$ at $90 \%$ CL is used to be comparable with direct detection experiments.

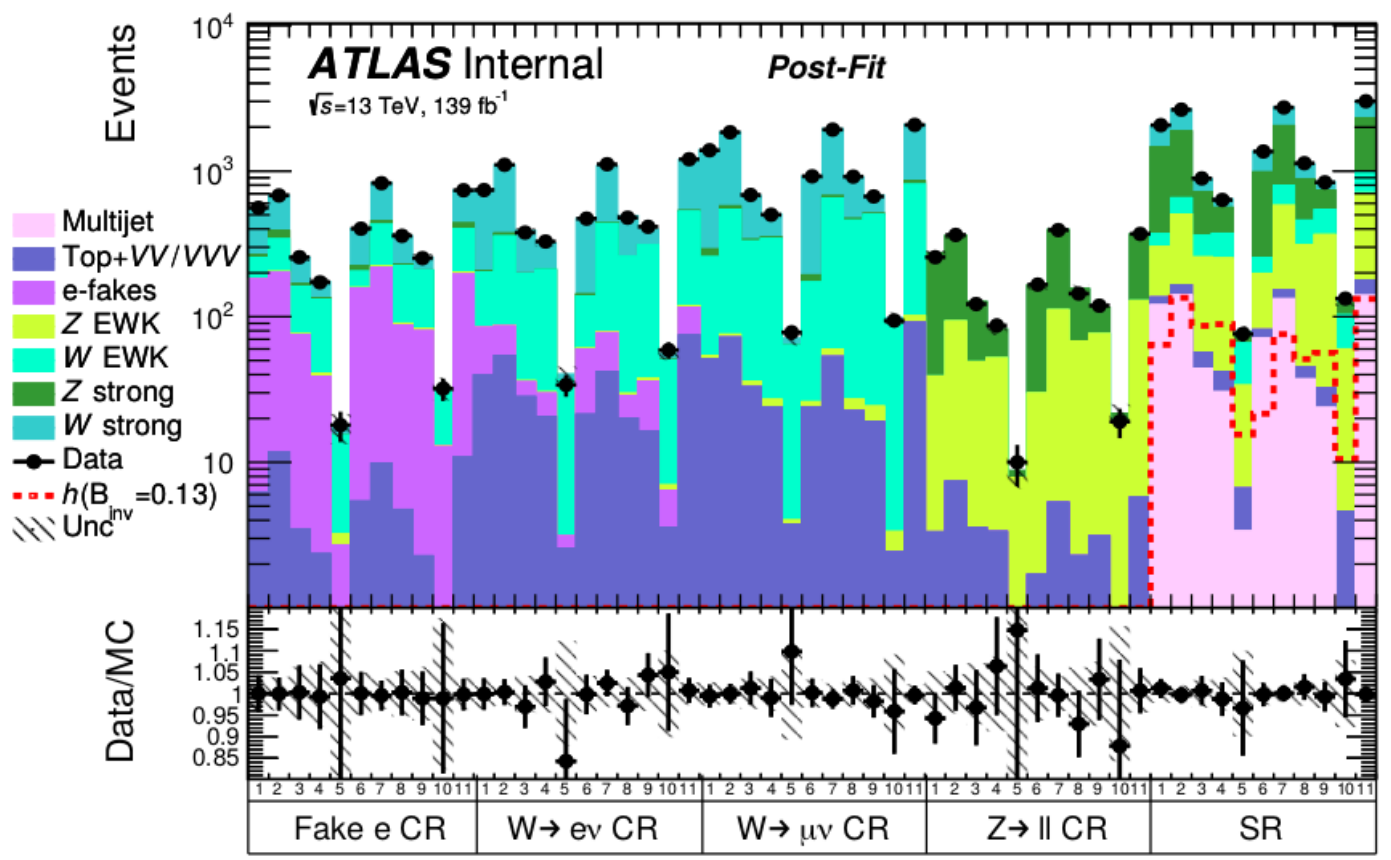

FIGURE 10: Postfit results of all SR and CR bins. The signal is scaled to a branching ratio of the Higgs boson into invisible final states of $13 \%$. bins in the SR and each of the CRs are defined in Fig 3

\section{INVISIBLE HIGGS COMBINATIONS}

This VBF analysis is used as input for a statistical combination of the Run 2 searches Ref.[6] for invisible decays of the Higgs boson with the $t \bar{t} \mathrm{H}$ topologies, considering separately the two and zero lepton final state channels. Also a statistical combination with the combined Run 1 results is performed by the ATLAS collaboration (see Fig 13 ).

No excess is observed above the standard model deviation and a new value of $11 \%$ at $95 \%$ CL is set for the limit on the invisible Higgs boson branching ratio. These combination are interpreted as a search for WIMP dark matter particles (see Fig 14 .

\section{CONCLUSION}

This analysis present a search for Higgs boson produced via the VBF mechanism and decay into invisible particles using a luminosity of $139 \mathrm{fb}-1$ of proton-proton collision at center of mass $\sqrt{s}=13 \mathrm{TeV}$ collected during the Run2 period using the ATLAS 


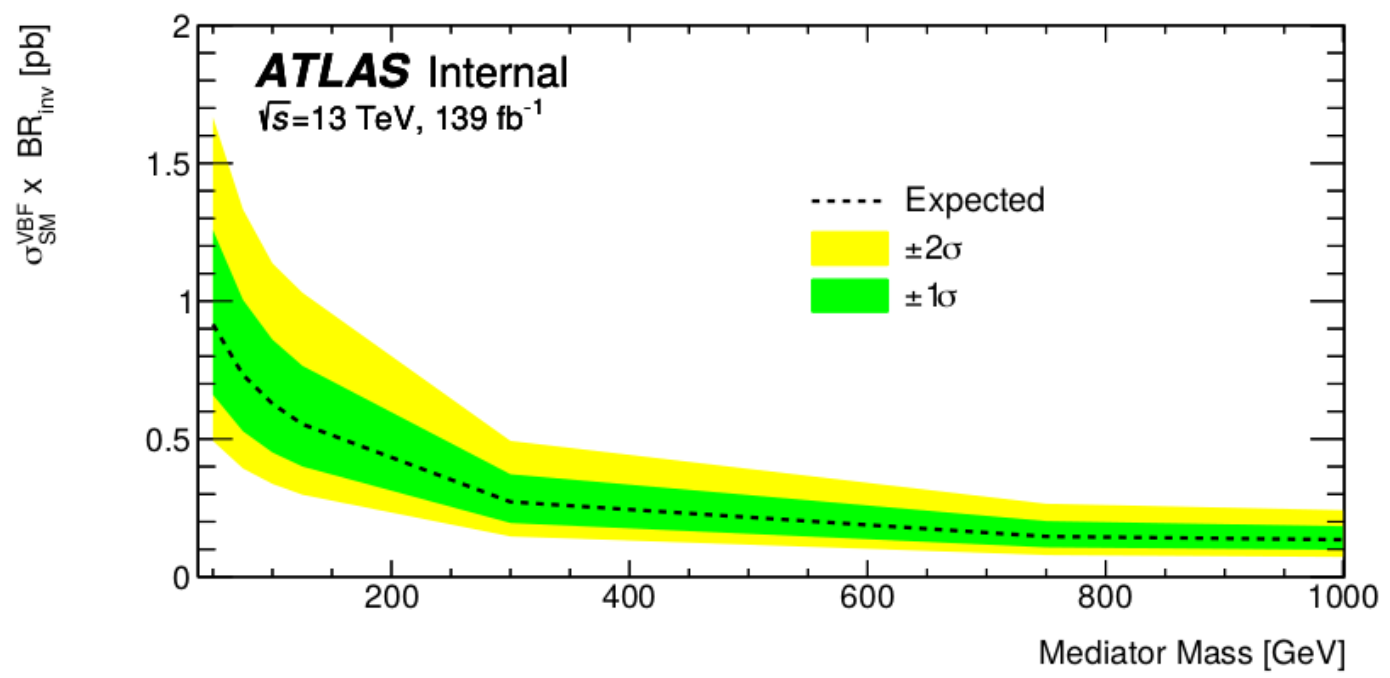

FIGURE 11: Upper limit on cross section times branching ratio to invisible particles for heavy scalar mediator particle as a function of its mass.

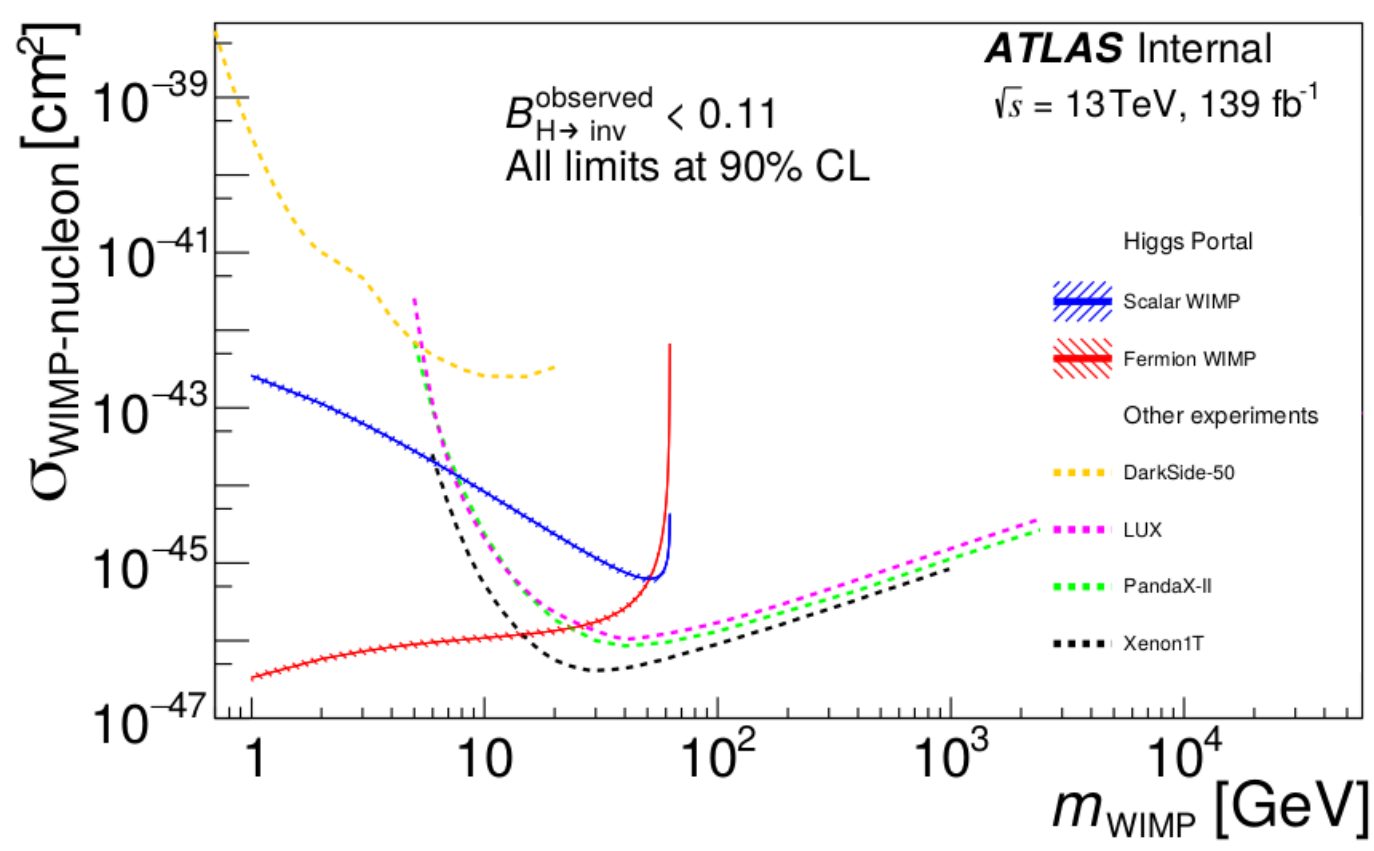

FIGURE 12: Comparison of the the observed exclusion limits from this analysis, to the upper limits from direct detection experiments on the spin-independent WIMP-nucleon scattering cross section, as a function of the WIMP mass [7, 8, 9, 10]. ATLAS results interpretation assumes Higgs portal scenarios where the $125 \mathrm{GeV}$ Higgs boson decays to a pair of DM particles that are either scalars or Majorana fermions. The hatched band represent the uncertainties from the nuclear form factor. The regions above the limit contours are excluded.

detector at the LHC. The experimental signature from VBF process is two leading jets with large values on the difference of their pseudo-rapidity $\left(\Delta \eta_{j j}\right)$ and large invariant masses $m_{j j}$ and $E_{T}^{m i s s}$. Events with three or four jets are considered as well if they originate from initial or final state radiations. An upper limit of 0.13 at $95 \%$ CL is set on $B_{H \rightarrow i n v}$ assuming the Standard Model Higgs boson production. Obtained results are interpreted as a search for invisible decays of a new scalar particles with masses of up to 1 $\mathrm{TeV}$ resulting in a limit on $\sigma^{V B F} \times B_{H \rightarrow i n v}$ of around $1 \mathrm{pb}$ at a mass of $50 \mathrm{GeV}$ and of $0.12 \mathrm{pb}$ for masses above $1 \mathrm{TeV}$. This result is interpreted using Higgs portal models to exclude regions in the $\sigma_{W I M P-n u c l u o n}$ parameter vs the WIMP mass parameter space. The statistical combination of this result with $t \bar{t} H$ topologies and with results from the LHC Run1 (H inv) combination, yields to a limit of 0.11 at $95 \%$ CL on the $B_{H \rightarrow i n v}$. 


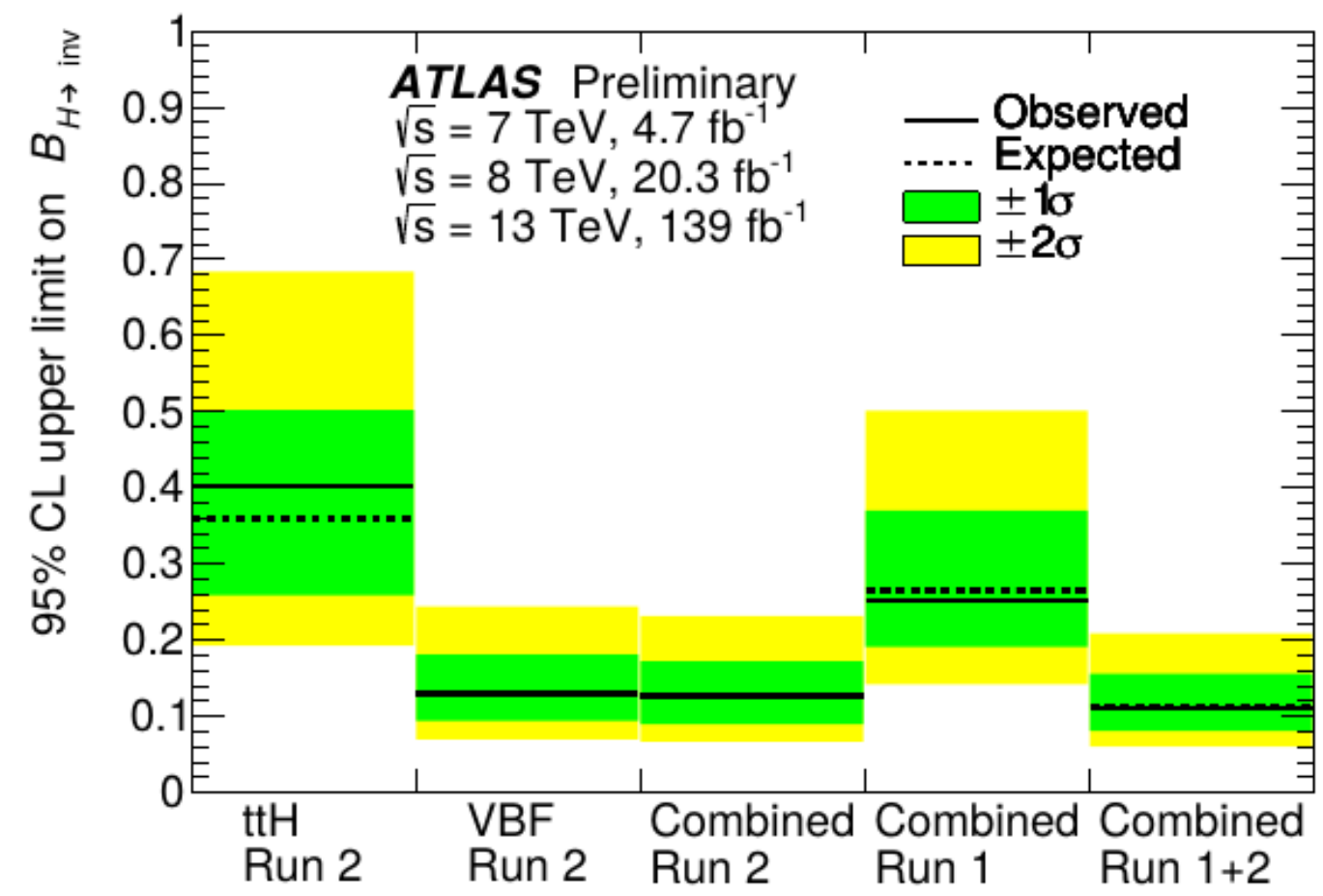

FIGURE 13: The observed and expected upper limits on $B_{H \rightarrow i n v}$ at the $95 \%$ CL from direct searches for invisible decays of the 125 $\mathrm{GeV}$ Higgs boson and their statistical combinations in Run 1 and 2.

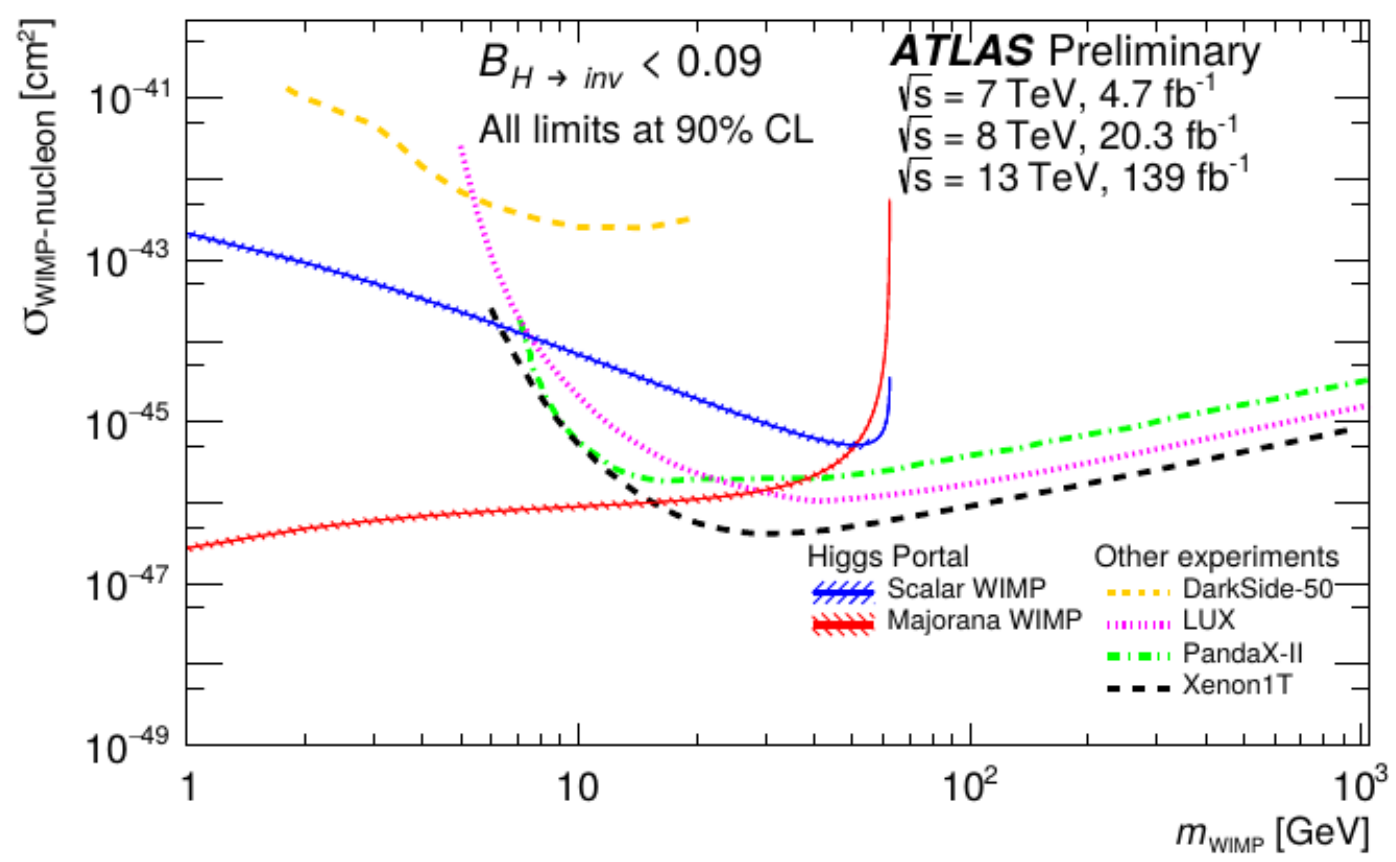

FIGURE 14: Comparison of the the observed exclusion limits from the combination to the upper limits from direct detection experiments on the spin-independent WIMP-nucleon scattering cross section, as a function of the WIMP mass. ATLAS results interpretation assumes Higgs portal scenarios where the $125 \mathrm{GeV}$ Higgs boson decays to a pair of DM particles that are either scalars or Majorana fermions. The hatched band represent the uncertainties from the nuclear form factor. The regions above the limit contours are excluded. 


\section{References}

[1] article Dark Matter: Evidence, Candidates and Constraints

[2] Handbook of LHC Higgs Cross Sections: 4. Deciphering the Nature of the Higgs Sector

[3] Status of invisible Higgs decays

[4] Search for invisible Higgs boson decays with vector boson fusion signatures with the ATLAS detector using an integrated luminosity of 139 $f b^{1}$.

[5] Search for invisible Higgs boson decays in vector boson fusion at $\sqrt{s}=13 \mathrm{TeV}$ with the ATLAS detector.

[6] Combination of searches for invisible Higgs bosondecays with the ATLAS experiment

[7] Results from a Search for Dark Matter in the Complete LUX Exposure

[8] Dark Matter Results From 54-Ton-Day Exposure of PandaX-II Experiment

[9] Dark Matter Search Results from a One TonnexYear Exposure of XENON1T

[10] Low-Mass Dark Matter Search with the DarkSide-50 Experiment 